\title{
Single Sitting Revitalization of Nonvital Young Permanent Teeth
}

\author{
Avninder Kaur ${ }^{1}$ Anchal Soni ${ }^{1}$ Harees Shabir ${ }^{1}$ \\ ${ }^{1}$ Department of Paediatric and Preventive Dentistry, Bhojia Dental \\ College and Hospital, Baddi, Himachal Pradesh, India
}

\begin{abstract}
Address for correspondence Avninder Kaur, MDS, Bhojia Dental College and Hospital, Baddi 173205, Himachal Pradesh, India (e-mail: avninder21@yahoo.com).
\end{abstract}

\begin{abstract}
Keywords

- nonvital teeth

- young permanent teeth

- revitalization

A 9-year-old child reported with chief complaint of broken teeth (11 and 21) and discolored tooth (21). Diagnosis of pulp necrosis with apical periodontitis with regard to 11 and 21 was made based on clinical and radiographic examination. The canals were irrigated with sodium hypochlorite and then dried with paper points. Disinfection of canals was done using photoactivated disinfection (PAD). Platelet-rich fibrin (PRF) was placed as scaffold in canals till the cementoenamel junction (CEJ). A 2-mm thick layer of white MTA was placed, followed by dual seal using glass-ionomer cement (GIC) and composite resin. Follow-up examination was done for 1 year. Clinical examination showed no pain, tenderness on percussion, and no mobility and improvement in color of teeth. Radiographic evaluation revealed continued thickening of the dentinal walls, root lengthening, regression of the periapical lesion, and partial apical closure. This report of pulp revascularization shows that disinfection with photodynamic therapy combined with PRF resulted in satisfactory root development in necrotic immature teeth.
\end{abstract}

\section{Introduction}

Endodontic treatment of nonvital young permanent teeth is a challenging proposition for the pediatric dentist. ${ }^{1}$ Difficulty in achieving an apical seal and thin dentinal walls, making biomechanical preparation difficult, are the main challenges. Also, the root crown ratio is unfavorable. Therefore, considering these factors, modern dentistry is shifting toward more biologically based treatment modalities for nonvital teeth.

The literature shows that the apical papilla in necrosed young permanent teeth has the potential to regenerate, if disinfected properly. By revitalization of pulp, the desired results can be achieved. The success of revitalization depends upon the following: disinfection of root canal, matrix in the canal for vital tissue growth, and tight coronal seal. ${ }^{2}$ Autologous matrices used for revitalization are as follows: blood clot formed by over instrumentation in periapical area, platelet-rich plasma (PRP) and platelet-rich fibrin (PRF).

Photoactivated disinfection (PAD) of the root canal is one of the recent advances in the field of regenerative endodontics where disinfection is achieved using laser irradiation. It makes revitalization a single-sitting procedure by placing the scaffold in the same visit. The purpose of this case report is to evaluate the efficacy of PAD and PRF in revitalization of a tooth with necrotic pulp and open apex over a single visit of the patient.

\section{Case Report}

A 9-year-old boy reported to the department of pediatric and preventive dentistry with broken teeth 11 and 21 (-Fig. 1). There was history of fall one-and-a-half years ago. Clinical examination revealed fracture of 11 and 21, involving enamel, dentin and pulp. Discoloration was seen in 21. No sensitivity to percussion or tenderness on palpation was observed. Periodontal examination revealed physiologic mobility and normal probing depth. The teeth were nonresponsive to the electric pulp test. Radiographic examination showed immature roots with open apices, and periapical radiolucencies in 11 and 21 (-Fig. 2). The final diagnosis involved pulp necrosis with apical periodontitis in relation to 11 and 21 . Considering the condition of pulp and periapical pathology
DOI https://doi.org/ 10.1055/s-0040-1714157 ISSN 2321-1482.
License terms

(요 (1) $\Theta \circledast$ 


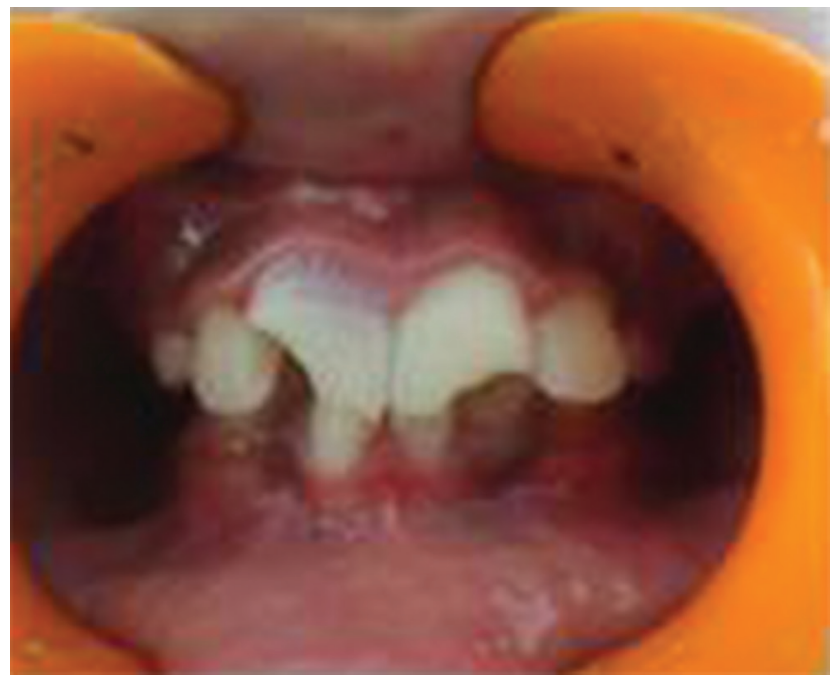

Fig. 1 Clinical appearance of 11, 21 before treatment.

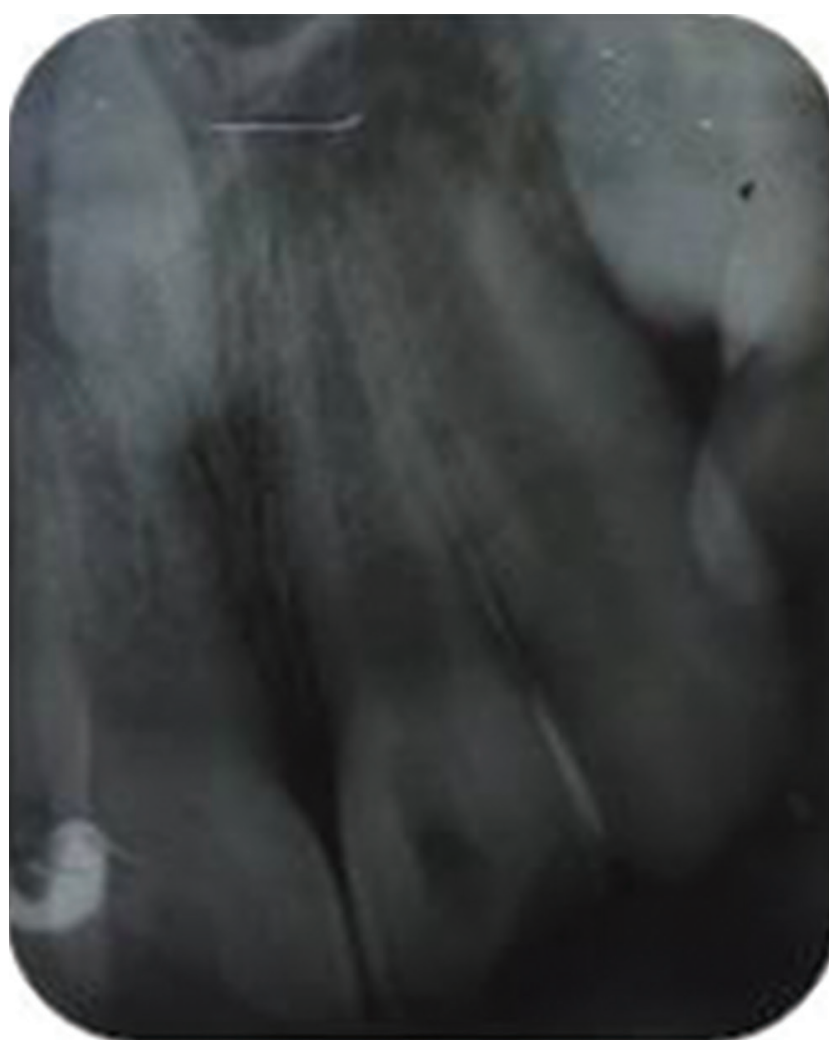

Fig. 2 Radiograph showing open apex of 11 and 21.

and status of root development, revitalization using PRF was planned. After explaining the procedure and its outcomes, a written consent was obtained from parents.

After isolation of teeth, disinfection of crown and surrounding rubber dam was done and root canal access was obtained without the use of local anesthesia. Straight line access was obtained, and working length was checked using size $80 \mathrm{~K}$ file and kept $2 \mathrm{~mm}$ short of radiographic apex. Minimal mechanical instrumentation was done to remove necrosed tissue and debris from the root canals. Copious irrigation with 3\% sodium hypochlorite was done using syringe and side vented needle, followed by sterile saline to remove necrotic tissues. A subsequent irrigation with 17\% EDTA followed by normal saline was done. The canal walls were dried using paper points. Before starting the disinfection of the root canal with LASER, PRF preparation was started. The patient's whole venous blood $(5 \mathrm{~mL})$ was collected by venipuncture of right antecubital vein with 22 -gauge needle and $10 \mathrm{~mL}$ syringe. Blood was immediately transferred into a sterile $10 \mathrm{ml}$ test tube without anticoagulant and centrifuged using tabletop centrifuge (Remi Elktrotechnik Limited, India) at 3,000 rpm for 10 minutes. PRF clot was removed using sterile tweezer and separated from the underlying RBCs layer using scissors and then transferred into a sterile dappen dish.

While the PRF is being prepared, disinfection of the root canal was done by using photoactivated diode laser system (Biolase) for 30 to 60 seconds. After drying with sterile paper point, photosensitizer solution ( $6.25 \mu \mathrm{g} / \mathrm{mL}$ Methylene blue) was placed inside the root canal using a needle $(0.5 \mathrm{~mL})$ and left inside the root canal for 2 minutes. The illumination was performed using diode laser system $(810 \mathrm{~nm}$ wavelength, $2.5 \mathrm{~W}$ power). The fiber was placed in the canal; spiral movements were made from apical to cervical for equal diffusion of light inside canal lumen. These movements were repeated approximately 6 to 10 times per minute. PRF was obtained in the form of a membrane by squeezing out the fluids in the fibrin clot using gauze pieces and cutting it into comparatively smaller pieces using sterile scissors (-Fig. 3). After disinfection of root canals, PRF was carried into root canals using finger plugger till the apical end. PRF was confined just below the level of cementoenamel junction (CEJ). A 2-mm thick layer of white MTA (Angelus) was placed over the PRF scaffold, followed by a moist cotton pellet. The patient had to wait for 15 to 20 minutes for setting of MTA. Permanent restoration was placed on the same day with double seal of glass-ionomer cement (GIC) and composite resin.

The patient was followed-up at 6- and 12-month intervals for clinical and radiographic follow-up. Radiographic evaluation revealed continued thickening of the dentinal walls, root lengthening, regression of the periapical lesion, and partial apical closure ( - Fig. 4). Clinical evaluation revealed no pain or tenderness on percussion and improvement in color of

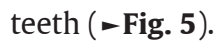

\section{Discussion}

Regenerative endodontic procedures are biologically based approaches to replenish the already damaged tooth structure. Young permanent teeth show favorable outcome because of wide open apical foramen. The other less favorable approaches are apexification and endodontic therapy. Conventional root canal treatment (RCT) is not possible in these teeth due to open apex and thin dentinal walls, which make tooth more vulnerable to fracture. Root end closure is achieved by apexification but with less favorable outcomes. Root crown ratio does not get altered after apexification. No change in pulp-dentin complex after 


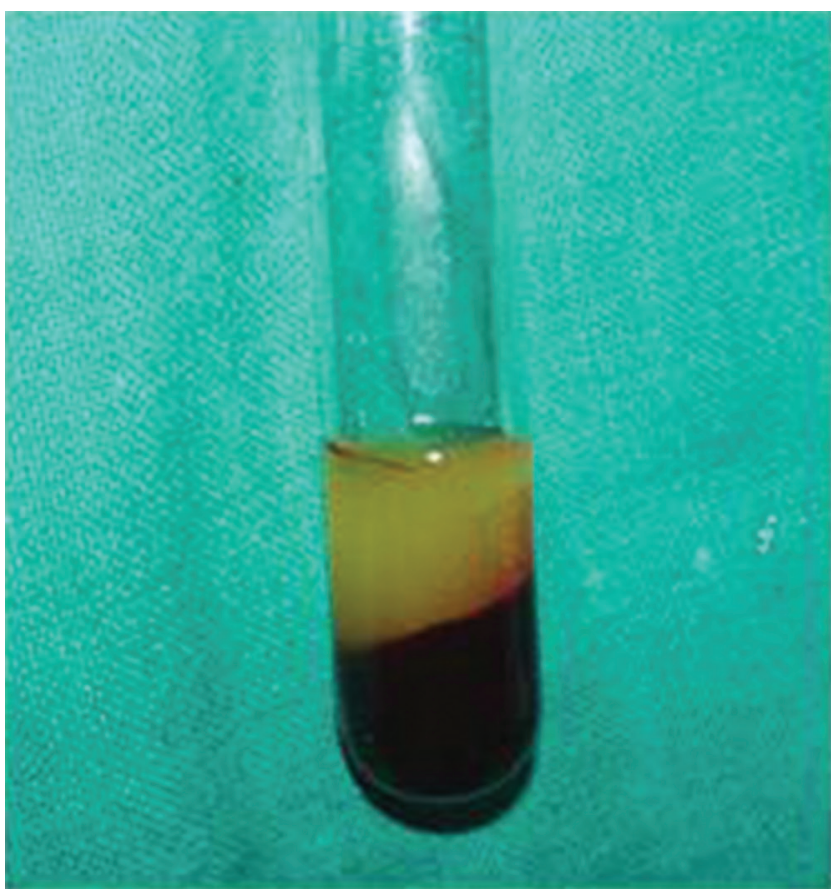

Fig. 3 Layers obtained after centrifugation.

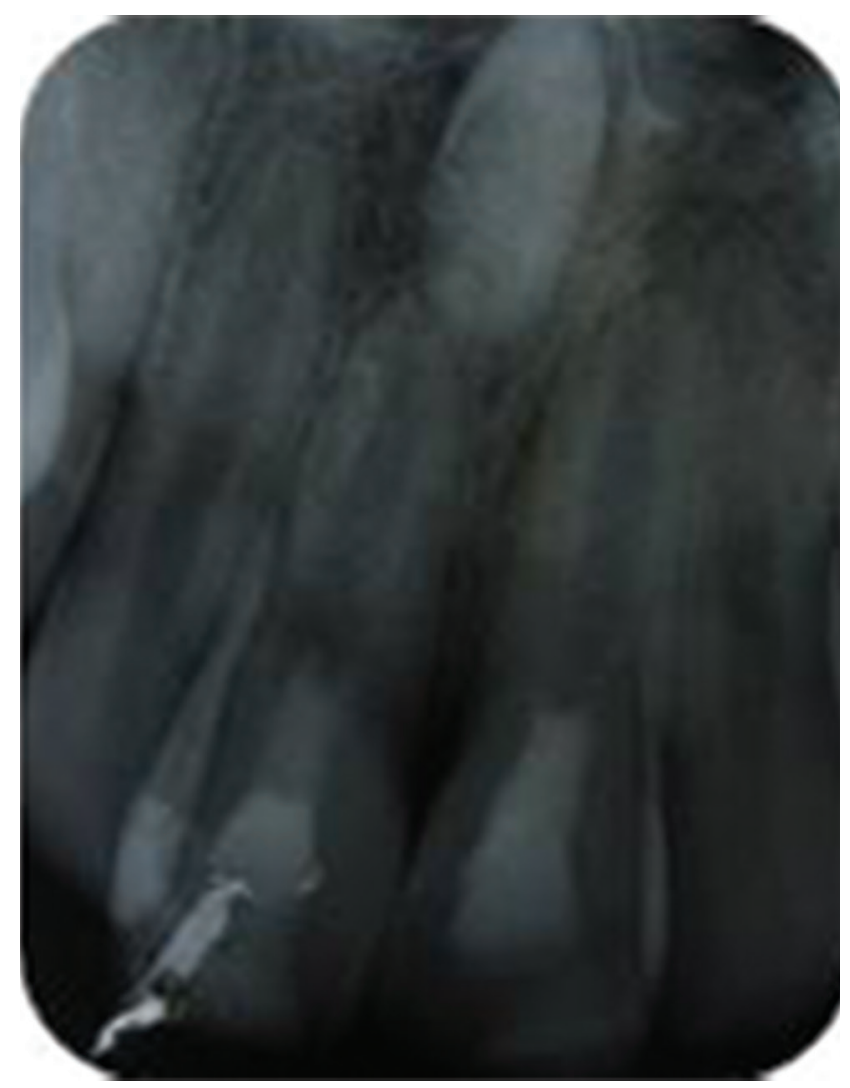

Fig. 4 Radiographic picture at 12-month follow-up.

apexification is seen; thus, revitalization is the treatment of choice in these cases.

The three components contributing to the success of the revitalization are stem cells, signaling molecules, and scaffold. ${ }^{3}$ PRF was selected as scaffold, as it overcomes

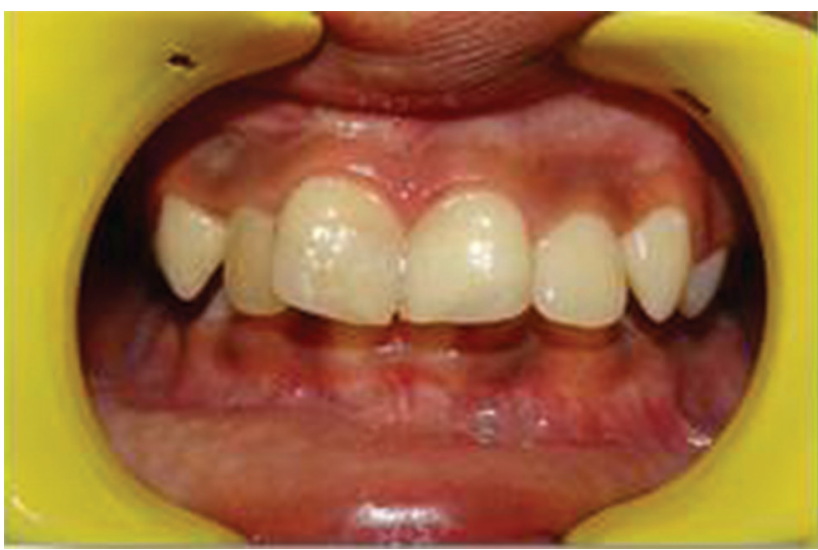

Fig. 5 Clinical picture at 12-month follow-up.

many drawbacks associated with blood clot and PRP. Instrumentation beyond the apical area may cause pain and discomfort to the patient, and blood clot formed in the canal space may not possess adequate strength matrix for carrying growth factors. ${ }^{4}$ Preparation of PRF is a single-step procedure without addition of anticoagulant (bovine thrombin), which makes it safer than PRP. It minimizes the chances of hemorrhage, thrombosis, and substantial immune reactions such as systemic lupus erythematosus (SLE). Also, PRF matrix contains higher concentration of cytokines as compared with PRP and blood clot. Healing kinetics is stronger and more consistent in PRF than PRP. ${ }^{5}$ PRF shows strong proliferation and differentiation of bone mesenchymal stem cells (BMSC) as compared with PRP. No associated cytotoxicity toward dental pulp stem cells, periadipocytes, dermal prekeratinocytes, osteoblasts, oral epithelial cells, periodontal ligament cells, and gingival fibroblasts has been shown by PRF. ${ }^{6}$ Handling properties of PRF are better as compared with PRP. Thus, PRF is a preferable scaffold in a clinical settings.

The successful outcome of any endodontic procedure is based on the efficient disinfection of the root canal system and prevention of reinfection. Many reported cases of revitalization procedures used triple antibiotic paste to disinfect the canals. In other cases, calcium hydroxide proved to be equally efficacious as an antibiotic in promoting root lengthening and thickening. The possible adverse effects of antibiotics include coronal discoloration, bacterial resistance, and allergic reactions. The high $\mathrm{pH}$ can also cause necrosis of multipotent stem cells on contact with it.

The most common reason for reinfection is improper disinfection and inadequacy of conventional irrigation solutions to cleanse root canals completely. Microorganisms associated with reinfection of root canal and endodontic treatment failures are Enterococcus faecalis and Candida albicans. ${ }^{7.8}$ Sodium hypochlorite is a potent antimicrobial agent, killing most bacteria instantly on direct contact. It also effectively dissolves vital and necrotic pulp remnants and collagen. However, there is evidence that hypochlorite is not effective against all pathogenic bacteria, such as $E$. faecalis and Candida albicans, which are associated with reinfected canals. 
To overcome these limitations, PAD was used in this case. Its antimicrobial strategy involves the combination of a nontoxic photosensitizer (PS) and a low-power laser to disinfect the root canal. ${ }^{9}$ The photosensitizers have a high-degree of selective killing microorganisms without affecting host cell viability. ${ }^{10}$

Apical closure and increasing radicular thickness were noted at the 12-month follow-up in both the central incisors. Improvement in sensibility test readings and restoration of the natural color of teeth were evident. This report of pulp revascularization shows that disinfection with photodynamic therapy (PDT) combined with PRF leads to satisfactory root development in necrotic immature teeth.

\section{Conclusion}

It was concluded that revitalization procedure using PRF and PAD has a predictable outcome and can surely be recommended as a single-sitting revitalization procedure in nonvital young permanent teeth.

\section{Conflict of Interest}

None declared.

\section{References}

1 Alagl A, Bedi S, Hassan K, AlHumaid J. Use of platelet-rich plasma for regeneration in non-vital immature permanent teeth: Clinical and cone-beam computed tomography evaluation. J Int Med Res 2017;45(2):583-593

2 Windley W II, Teixeira F, Levin L, Sigurdsson A, Trope M. Disinfection of immature teeth with a triple antibiotic paste. J Endod 2005;31(6):439-443
3 Hargreaves KM, Giesler T, Henry M, Wang Y. Regeneration potential of the young permanent tooth: what does the future hold? J Endod 2008;34(7(Suppl):S51-S56

4 Shivashankar VY, Johns DA, Vidyanath S, Kumar MR. Platelet rich fibrin in the revitalization of tooth with necrotic pulp and open apex. J Conserv Dent 2012;15(4):395-398

5 Dohan DM, Choukroun J, Diss A, et al. Platelet-rich fibrin (PRF): a second-generation platelet concentrate. Part I: technological concepts and evolution. Oral Surg Oral Med Oral Pathol Oral Radiol Endod 2006;101(3):e37-e44

6 Torabinejad M, Ung B, Kettering JD. In vitro bacterial penetration of coronally unsealed endodontically treated teeth. J Endod 1990;16(12):566-569

7 Siddiqui SH, Awan KH, Javed F. Bactericidal efficacy of photodynamic therapy against Enterococcus faecalis in infected root canals: a systematic literature review. Photodiagn Photodyn Ther 2013;10(4):632-643

8 Mohan D, Maruthingal S, Indira R, et al. Photoactivated disinfection (PAD) of dental root canal system - An ex-vivo study. Saudi J Biol Sci 2016;23(1):122-127

9 Demidova TN, Hamblin MR. Photodynamic therapy targeted to pathogens. Int J Immunopathol Pharmacol 2004;17(3):245-254

10 Lee MT, Bird PS, Walsh LJ. Photo-activated disinfection of the root canal: a new role for lasers in endodontics. Aust Endod J 2004;30(3):93-98 